\title{
Macrofauna edáfica em lavouras de cana-de-açúcar e mata no noroeste do Paraná - Brasil
}

\section{Edaphic macrofauna in sugar cane crops and forest in northwestern Paraná - Brazil}

\author{
Luiz Angelo Pasqualin ${ }^{1 *}$; Jair Alves Dionísio; \\ Maria Aparecida Cassilha Zawadneak ${ }^{2}$; Caroline Teixeira Marçal ${ }^{1}$
}

\begin{abstract}
Resumo
O objetivo deste trabalho foi caracterizar a macrofauna edáfica e avaliar os efeitos ocasionados em virtude do método de colheita e aplicação de vinhaça em áreas de cana-de-açúcar comparativamente com uma área de mata. $\mathrm{O}$ delineamento experimental foi inteiramente casualizado, com cinco tratamentos (colheita manual com uso do fogo e mecanizada sem uso do fogo associadas ou não à aplicação de vinhaça em adubação, e mata) e cinco repetições durante seis épocas amostrais. Retiraram-se monólitos de $25 \mathrm{~cm}$ x $25 \mathrm{~cm}$ x $30 \mathrm{~cm}$, sendo os organismos do solo coletados e classificados em grupos e/ou ordem. A caracterização dos grupos nos tratamentos foi realizada por análise de componentes principais. Após o cálculo da densidade de organismos, dos índices ecológicos de diversidade (Shannon), equitabilidade (Pielou) e riqueza de grupos, os valores foram comparados através do teste Duncan ou Kruskal-Wallis a 5\%. Comparativamente com as áreas de cana-de-açúcar, a área de mata apresenta maiores valores dos índices calculados. Os tratamentos de cana-de-açúcar com manutenção da cobertura e/ou aplicação de vinhaça favorecem a fauna edáfica.
\end{abstract}

Palavras-chave: Saccharum officinarum, fauna edáfica, palhada, vinhaça, mata

\begin{abstract}
The objective of this study was to characterize the soil fauna and assessing the effects of harvest method and vinasse application in areas of sugar cane compared to a forest area. The experimental design was completely randomized with five treatments (manual harvesting with use of fire and mechanical harvesting without use of fire associate to or not vinasse application fertilizer and forest), five repetitions for six sampling times. Retired monoliths $25 \mathrm{~cm}$ x $25 \mathrm{~cm}$ x $30 \mathrm{~cm}$, where soil organisms were sorted and classified into groups and /or order. The characterization of the treatments was performed by principal component analysis. Having previously calculated the density of organisms, the ecological indices of diversity (Shannon), evenness (Pielou) and richness of groups, these were compared by Duncan or Kruskal-Wallis test at $5 \%$. Compared with the areas of sugar cane, the forest area has the highest calculated indices. The treatments of sugar cane with maintenance of straw and /or vinasse application favor soil fauna.
\end{abstract}

Key words: Saccharum officinarum, soil fauna, straw, vinasse, forest

1 Discentes da Universidade Federal do Paraná, UFPR. Curitiba, PR. E-mail: luiz.pasqualin@seab.pr.gov.br; carolinemarcal@ seab.pr.gov.br

2 Profs.da UFPR, Curitiba, PR. E-mail: jair@ufpr.br; mazawa@ufpr.br

* Autor para correspondência 


\section{Introdução}

O Brasil é o maior produtor mundial de canade-açúcar, seguido por Índia, Tailândia e Austrália, ocupando o Estado do Paraná a segunda posição na produção nacional (CONAB, 2005).

O método de colheita da cultura vem gradualmente mudando do sistema tradicional, onde se realiza a queima prévia e corte manual, para o sistema mecanizado, realizado sem uso do fogo, principalmente em virtude de exigências legais. Pesquisas apontam benefícios da manutenção da palhada sobre as características físicas e químicas do solo (CANELLAS et al., 2007), produtividade do canavial (SOUZA et al., 2005), além de impactos negativos das queimadas sobre as populações e trabalhadores locais (RIBEIRO, 2008; URIARTE et al., 2009).

A vinhaça, principal resíduo da destilação do álcool, vem recebendo atenção devido ao seu potencial nutricional, sendo as pesquisas voltadas principalmente para os efeitos sobre as plantas (GÓMES; RODRIGUEZ, 2000; AZANIA et al., 2003; CANELLAS et al., 2003; SANOMIYA et al., 2006).

A qualidade do solo, sendo entendida como o equilíbrio entre os condicionantes geológicos, hidrológicos, químicos e biológicos (SPOSITO; ZABEL, 2003) pode desempenhar um importante papel na sustentabilidade do setor sucroalcooleiro.

De modo geral os resultados de trabalhos onde se comparam sistemas agrícolas e naturais mostram que a macrofauna é fortemente afetada pela ação antrópica. Em estudo do efeito do manejo agrícola sobre minhocas na África tropical, Dlamini e Hayanes (2004) constataram maior número de indivíduos em áreas de mata, seguido de cana colhida sem queima e em último lugar com queima. Pinheiro, Santos e Garay (1996), em trabalho onde se comparou a fauna edáfica em áreas de cana, encontraram redução no número de grupos nas áreas submetidas à queima.

Apesar de ser reconhecida a importância da macrofauna para o equilíbrio e funcionamento dos ecossistemas, os poucos estudos realizados sobre a macrofauna edáfica em áreas de cana de açúcar no Brasil restringem-se às regiões tropicais, sendo que a atual expansão canavieira para regiões subtropicais merece atenção.

Este trabalho teve como objetivo caracterizar a macrofauna edáfica e avaliar os efeitos do método de colheita e aplicação de vinhaça em áreas de canade-açúcar comparativamente a uma área de mata durante um ano.

\section{Material e Métodos}

$\mathrm{O}$ experimento foi instalado em lavoura da usina de álcool e açúcar Alto Alegre S.A, localizada no município de Colorado - Paraná, situado à 22 $2^{\circ} 50^{\prime} 15^{\prime}$ " S e $51^{\circ} 58^{\prime} 23$ " W, e altitude de 400 metros, sobre Latossolo Vermelho distrófico (EMBRAPA, 2006), textura arenosa, fase floresta subtropical perenifólia e relevo plano a suave ondulado (Tabela 1). O clima regional é do tipo Cfa (subtropical úmido mesotérmico), possuindo verões quentes com tendência de concentração das chuvas (temperatura média superior a $22^{\circ} \mathrm{C}$ ), invernos com geadas pouco freqüentes (temperatura média inferior a $18^{\circ} \mathrm{C}$ ). A precipitação média anual varia de 1400 a $1600 \mathrm{~mm}$ e a mensal em torno de $104 \mathrm{~mm}$ (IAPAR, 1994). 
Tabela 1. Caracterização química média dos solos (seis repetições), na profundidade de $0-0,30 \mathrm{~cm}$, realizada na Usina Alto Alegre, Município de Colorado - Paraná, de novembro 2007 a setembro 2008.

\begin{tabular}{ccccccccccccc}
\hline Trat. & $\begin{array}{c}\mathrm{pH} \\
\mathrm{CaCl}_{2}\end{array}$ & M.O. & $\begin{array}{c}\mathrm{P} \\
\text { (resina) }\end{array}$ & $\mathrm{K}$ & $\mathrm{Ca}$ & $\mathrm{Mg}$ & $\mathrm{H}+\mathrm{Al}$ & $\mathrm{Al}$ & $\begin{array}{c}\text { Soma } \\
\text { bases }\end{array}$ & $\mathrm{CTC}$ & $\begin{array}{c}\text { Sat. } \\
\text { bases }\end{array}$ & $\begin{array}{c}\text { Sat. } \\
\mathrm{Al}\end{array}$ \\
\hline & & $\mathrm{g} \mathrm{dm}^{-3}$ & $\mathrm{mg} \mathrm{dm}^{-3}$ & & ----------------- & $\mathrm{mmol}_{\mathrm{c}} \mathrm{dm}^{-3}----------------$ & $\mathrm{V} \%$ & $\mathrm{~m} \%$ \\
\hline CPCV & 5,52 & 14,18 & 35,58 & 2,31 & 15,90 & 6,70 & 14,22 & 0,00 & 25,18 & 39,40 & 63,7 & 0 \\
SPCV & 5,69 & 15,87 & 124,01 & 2,99 & 19,06 & 6,46 & 14,89 & 0,00 & 28,49 & 43,38 & 66,0 & 0 \\
CPSV & 4,58 & 15,10 & 11,61 & 0,88 & 12,90 & 2,60 & 25,13 & 1,40 & 16,51 & 41,64 & 40,0 & 8 \\
SPSV & 4,28 & 13,83 & 16,81 & 1,32 & 10,39 & 2,11 & 30,43 & 3,28 & 13,90 & 44,33 & 31,3 & 21 \\
MT & 4,04 & 25,50 & 8,56 & 0,88 & 13,54 & 3,68 & 44,38 & 4,11 & 18,33 & 62,71 & 28,7 & 22 \\
\hline
\end{tabular}

Legenda: CPCV - com palha com vinhaça; SPCV - sem palha com vinhaça; CPSV - com palha sem vinhaça; SPSV - sem palha sem vinhaça; $\mathrm{MT}$ - mata.

O delineamento experimental foi inteiramente casualizado, tendo cinco tratamentos, sendo que cada um possuiu cinco repetições de um hectare, e de cada repetição foram retirados cinco monólitos, totalizando vinte e cinco monólitos em cada tratamento. Foram empregados os tratamentos: a) colheita mecanizada mantendo-se cobertura do solo e aplicação de vinhaça, denominado com palha e com vinhaça (CPCV); b) colheita mecanizada mantendo-se a cobertura do solo e sem aplicação de vinhaça, denominado com palha e sem vinhaça (CPSV); c) colheita manual com uso do fogo e aplicação de vinhaça, denominado sem palha e com vinhaça (SPCV); d) colheita manual com uso do fogo e sem aplicação de vinhaça, denominado sem palha e sem vinhaça (SPSV); e) mata sem interferência antrópica há 30 anos, denominado mata (MT).

Nos canaviais adotou-se espaçamento 1,40 x 0,40 $\mathrm{m}$ e todos os tratamentos avaliados encontravam-se "cana de segunda soca", ou seja, o rebrote de três cortes anteriores. No caso dos tratamentos em que a colheita é feita de forma mecanizada, a palhada já estava mantida há 36 meses. Nos tratamentos em que se utilizou vinhaça, esta foi aplicada nos canais, no volume de $120 \mathrm{~m}^{3} \mathrm{ha}^{-1}$ aos meses de novembro dos últimos três anos.

As amostragens da fauna edáfica foram realizadas de novembro de 2007 a setembro de 2008 a cada dois meses, pelo método Tropical Soil Biology and
Fertility (TSBF) (ANDERSON; INGRAM, 1993) modificado. Monólitos de 25 x $25 \mathrm{~cm}$ foram retirados na camada $0-10 \mathrm{~cm}$, incluindo a serrapilheira, e nas camadas 10-20 e 20-30 cm, por meio de um gabarito de madeira, colocado aleatoriamente na entrelinha da cultura a uma distância mínima de cinco metros. A macrofauna do solo presente em cada camada foi triada e preservada em álcool $70 \%$, sendo posteriormente identificada em grandes grupos ou ordens taxonômicas.

A densidade de organismos, os índices ecológicos de diversidade (Shannon), equitabilidade (Pielou) e riqueza de grupos, descritos por Baretta et al. (2003), foram comparadas através do teste Duncan a 5\%, quando seus valores foram normais por ShapiroWilk (p-0,05). Valores não normais mesmo após transformações foram comparados por KruskalWallis a 5\%. A média da densidade das classes ou ordens encontradas em cada época amostral foi submetida à Análise de Componentes Principais (ACP) através do software CANOCO versão 4.5 (TER BRAAK; SMILAURER, 2002).

\section{Resultados e Discussão}

Os valores médios da densidade da macrofauna edáfica variaram de 35,2 no tratamento SPSV em maio (2008) a 1.606,4 ind $\mathrm{m}^{-2}$ em janeiro (2008) na área de mata (Tabela 2). Comparado aos tratamentos com cana-de-açúcar, MT apresentou maior 
densidade de indivíduos da macrofauna edáfica, concordando com Lourente et al. (2007). Embora não tenham apresentado diferenças estatísticas, as áreas de cana-de-açúcar que receberam alguma fonte de matéria orgânica (palha, vinhaça ou ambos), mostraram tendência de maior densidade de organismo do que o tratamento SPSV. Em todos os tratamentos não houve diferença significativa quando avaliada a flutuação da densidade ao longo do tempo, resultados similares aos de Moço et al. (2005).

Tabela 2. Densidade da macrofauna edáfica $\left(\right.$ ind. $\mathrm{m}^{2}$ ) nas áreas da usina Alto Alegre, município de Colorado - Paraná, ao longo das seis épocas de coleta de novembro 2007 a setembro $2008^{(1)}$

\begin{tabular}{llllllll}
\hline Tratamento & Novembro & Janeiro & Março $^{\text {ns }}$ & Maio & Julho $^{\text {ns }}$ & Setembro & Média \\
\cline { 2 - 7 } CPCV $^{\text {ns }}$ & $259,2 \mathrm{~B}$ & $198,4 \mathrm{~B}$ & 182,4 & $256 \mathrm{~A}$ & 451,2 & $217,6 \mathrm{~B}$ & $259,2 \mathrm{~B}$ \\
CPSV $^{\text {ns }}$ & $99,2 \mathrm{BC}$ & $156,8 \mathrm{~B}$ & 144 & $192 \mathrm{AB}$ & 275,2 & $227,2 \mathrm{~B}$ & $181,9 \mathrm{~B}$ \\
SPCV $^{\text {ns }}$ & $54,4 \mathrm{BC}$ & $99,2 \mathrm{~B}$ & 131,2 & $259,2 \mathrm{~A}$ & 377,6 & $521,6 \mathrm{~A}$ & $240,5 \mathrm{~B}$ \\
SPSV $^{\text {ns }}$ & $57,6 \mathrm{C}$ & $204,8 \mathrm{~B}$ & 99,2 & $35,2 \mathrm{~B}$ & 220,8 & $201,6 \mathrm{~B}$ & $136,5 \mathrm{~B}$ \\
MT $^{\text {ns }}$ & $844,8 \mathrm{~A}$ & $1.606,4 \mathrm{~A}$ & 428,8 & $406,4 \mathrm{~A}$ & 336 & $422,4 \mathrm{AB}$ & $674,7 \mathrm{~A}$ \\
\hline Média $^{\text {ns }}$ & 263,04 & 452,48 & 197,12 & 229,76 & 330,88 & 318,88 & \\
\hline
\end{tabular}

(1) Médias de cinco repetições; valores na mesma época seguidos de letras iguais (maiúsculas) não se diferenciaram significativamente pelo teste de Duncan a 5\%; valores no mesmo tratamento seguidos de letras iguais (minúscula) não se diferenciaram significativamente pelo teste de Duncan a 5\%; CPCV: com palha e com vinhaça; CPSV: com palha e sem vinhaça; SPCV: sem palha e com vinhaça; SPSV: sem palha e sem vinhaça; MT: mata; ns: não significativo.

Figura 1. Densidades médias anuais (ind. $\mathrm{m}^{-2}$ ) distribuídas em grupos da macrofauna edáfica em áreas da Usina Alto Alegre, município de Colorado - Paraná, ao longo das seis épocas de coleta de novembro 2007 a setembro 2008. CPCV: com palha e com vinhaça; CPSV: com palha e sem vinhaça; SPCV: sem palha e com vinhaça; SPSV: sem palha e sem vinhaça; MT: mata. Médias de 6 repetições. Valores seguidos de letras iguais dentro do mesmo grupo não diferem pelo teste de Kruskal-Wallis a 5\%.

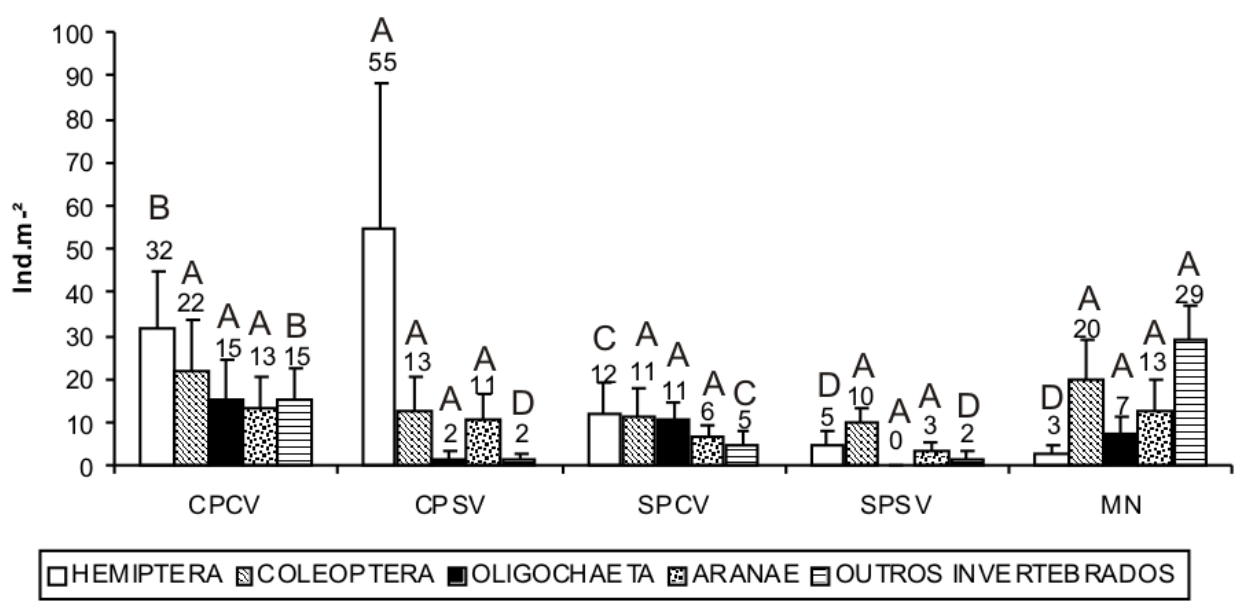

Fonte: Elaboração dos autores. 
A distribuição das densidades médias no ano por agrupamento em ordens e/ou grupos é expressa na Figura 1.

Optou-se em separar a comunidade de Hemiptera, Coleoptera, Oligochaeta, Araneae e Outros Invertebrados (composto por grupos com menor freqüência: Blattodea, Chilopoda, Diplopoda,
Diptera, Hymenoptera, Isopoda, Lepidoptera, Mollusca, Myriapoda, Orthoptera, Phasmatodea, Psocoptera e Symphyla), dos insetos sociais (Formicidae e Isoptera) (Figura 2). Tal medida foi necessária em virtude da característica destes dois grupos, que em razão das elevadas densidades, tornaram a escala gráfica desproporcional ao restante dos organismos coletados.

Figura 2. Densidades médias anuais (ind. $\mathrm{m}^{-2}$ ) distribuídas nos grupos Formicidae e Isoptera da macrofauna edáfica em áreas da usina Alto Alegre, município de Colorado - Paraná, ao longo das seis épocas de coleta de novembro 2007 a setembro 2008. CPCV: com palha e com vinhaça; CPSV: com palha e sem vinhaça; SPCV: sem palha e com vinhaça; SPSV: sem palha e sem vinhaça; MT: mata. Médias de 6 repetições. Valores seguidos de letras iguais dentro do mesmo grupo não diferem pelo teste de Kruskal-Wallis.

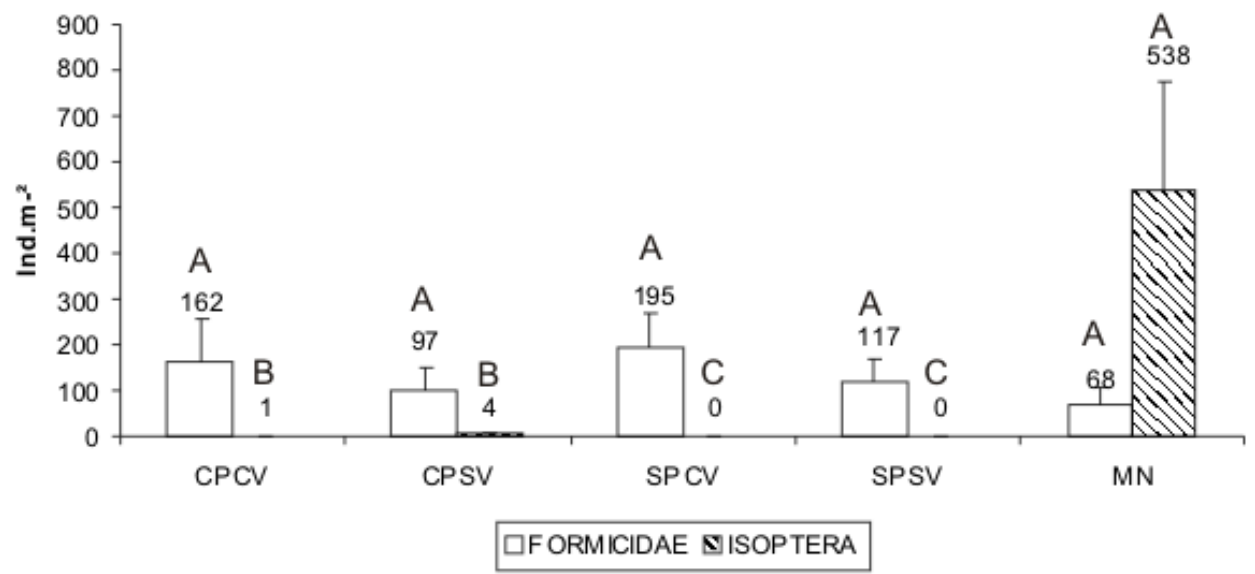

Fonte: Elaboração dos autores.

O grupo Hemiptera apresentou maior densidade em CPSV, sendo constituído de cochonilhas (Hemiptera, Coccidae), e principalmente por percevejos do gênero Scaptocoris (Hemiptera, Cydnidae) praga secundária em raízes de cana-deaçúcar, cujo nível de dano econômico ainda não foi estabelecido (NARDI, 2005).

A densidade do grupo Coleoptera não apresentou diferenças significativas entre os tratamentos.

Para o grupo Oligochaeta foram encontradas maiores densidades médias em $\mathrm{CPCV}$, seguido de SPCV e MT, embora estatisticamente não haja diferenciação. No tratamento CPSV foram encontrados apenas dois indivíduos no ano e em SPSV nenhum Oligochaeta foi encontrado. Resultados semelhantes foram encontrados por Nunes et al. (2006), onde áreas convertidas em canaviais manejados convencionalmente, reduziram as densidades e riquezas de minhocas encontradas. Ainda de acordo com estes autores, esta redução se daria pelo dano direto ocasionado pelos implementos, exposição a pássaros e inimigos naturais, condições de inibição de crescimento, desenvolvimento e reprodução, incluindo a redução da matéria orgânica, e capacidade hídrica do solo, quando da renovação dos canaviais.

A densidade do grupo Araneae, foi beneficiada pelos tratamentos CPCV, CPSV e MT, o que pode ser explicado pela manutenção da cobertura, que 
serve de abrigo e alimento para as aranhas e suas presas. Corroborando com estes resultados, os tratamentos SPCV e SPSV apresentaram as menores densidades. Embora o grupo Araneae possua uma maior capacidade de resiliência às modificações ambientais (PINHEIRO; SANTOS; GARAY, 1996), o ambiente de mata proporcionou melhores condições para este grupo. Como as aranhas são predadoras, a mata proporciona uma gama mais diversificada de possíveis presas (FLÓREZ, 2000) do que áreas de monocultura.

Os tratamentos com a manutenção da cobertura superficial foram os que apresentaram maiores densidades para os grupos classificados como Outros Invertebrados. A presença destes organismos pode ser sintetizada pelos benefícios proporcionados pela manutenção da cobertura, como maior umidade do solo e presença de matéria orgânica (COSTA; GOEDERT; SOUSA, 2006).

Nas áreas de cana, o grupo Formicidae foi o predominante, sendo que na área de mata, os cupins (Isoptera) sempre mantiveram dominância, sendo responsáveis pela diferenciação em termos de densidade. Estes resultados concordaram com os apresentados por Bianchi, Aquino e Almeida (2007), que também encontraram maior densidade de cupins em área de mata. Contudo, diferentemente dos resultados destes autores, o grupo Formicidae foi dominante no tratamento CPCV. Anderson et al. (2002) citam que elevada presença de formigas estão comumente associadas a alterações no manejo do solo, especialmente durante recondicionamento do solo, o que explicaria seus menores valores na área de mata em comparação com áreas de cana.

Embora os resultados demonstrem apenas uma tendência, as densidades dos grupos da macrofauna nas áreas de cana, sugerem benefícios em função da aplicação da vinhaça e manutenção da palhada. Estes resultados podem ser explicados em função das melhores condições de fertilidade e pH proporcionados pela vinhaça (SILVA et al., 2006), e pela matéria orgânica fornecida pela cobertura (RAZAFIMBELO et al., 2006).

Os resultados da avaliação da diversidade, expressos através do índice de Shannon, são expostos através da Tabela 3. A análise estatística entre os tratamentos mostrou que apenas nos meses de maio e julho ocorreram diferenças significativas entre os tratamentos. Os índices calculados apresentaramse relativamente baixos em função da dominância de Formicidae e Isoptera, tendo o mesmo sido observado por Lima et al. (2007) no estudo de sistemas agroflorestais. Os autores encontraram ainda que o tratamento com uso de fogo apresentou os mais baixos índices de diversidade, assim como os resultados observados neste estudo.

Tabela 3. Diversidade da macrofauna edáfica (índice de diversidade de shannon) nas áreas da usina Alto Alegre, município de Colorado - Paraná, ao longo das seis épocas de coleta de novembro 2007 a setembro $2008^{(1)}$.

\begin{tabular}{|c|c|c|c|c|c|c|c|}
\hline Tratamentos & Novembro ${ }^{\mathrm{ns}}$ & Janeiro $^{\text {ns }}$ & Marçons & Maio & Julho & Setembro ${ }^{\text {ns }}$ & Média $^{\text {ns }}$ \\
\hline $\mathrm{CPCV}^{\mathrm{ns}}$ & 0,1565 & 0,4532 & 0,2559 & $0,3389 \mathrm{AB}$ & $0,4608 \mathrm{AB}$ & 0,5377 & 0,3672 \\
\hline $\mathrm{CPSV}^{\mathrm{ns}}$ & 0,3289 & 0,2419 & 0,1941 & $0,2177 \mathrm{AB}$ & $0,4715 \mathrm{AB}$ & 0,6371 & 0,3485 \\
\hline$S P C V^{n s}$ & 0,3563 & 0,3105 & 0,2710 & $0,1305 \mathrm{BC}$ & $0,1072 \mathrm{C}$ & 0,4401 & 0,2693 \\
\hline SPSV $^{(2)}$ & $0,2955 \mathrm{~b}$ & $0,1012 \mathrm{c}$ & $0,0602 \mathrm{~d}$ & $0,0303 \mathrm{C} \mathrm{d}$ & $0,3786 \mathrm{~B}$ a & $0,3663 \mathrm{a}$ & 0,2054 \\
\hline $\mathrm{MT}^{\mathrm{ns}}$ & 0,3183 & 0,3575 & 0,5203 & $0,3993 \mathrm{~A}$ & $0,6401 \mathrm{~A}$ & 0,4379 & 0,4456 \\
\hline Média $^{\text {ns }}$ & 0,2911 & 0,2929 & 0,2603 & 0,2233 & 0,4116 & 0,4838 & \\
\hline
\end{tabular}

${ }^{(1)}$ Médias de cinco repetições. Análise sobre dados transformados por raiz $(\mathrm{x}+1)$; valores na mesma época seguidos de letras iguais (maiúsculas) não se diferenciaram significativamente pelo teste de Duncan a 5\%. ${ }^{(2)}$ Valores no mesmo tratamento seguidos de letras iguais (minúscula) não se diferenciaram significativamente pelo teste de Kruskal-Wallis a 5\%. CPCV: com palha e com vinhaça; CPSV: com palha e sem vinhaça; SPCV: sem palha e com vinhaça; SPSV: sem palha e sem vinhaça; MT: mata; ns: não significativo. 
Para o mês de maio, o índice de diversidade do tratamento SPSV foi significativamente menor que todos os outros. Segundo Souza et al. (2005) a maior exposição do solo a perda de umidade, maior variação de temperatura e menor aporte de alimento para alguns grupos, em consequência da eliminação da liteira, leva a diminuição do número de organismos mais sensíveis, reduzindo a diversidade. Os demais tratamentos tiveram médias semelhantes, porém mostrando leve tendência da cobertura do solo de elevação dos índices de Shannon.

$\mathrm{Na}$ coleta de julho, as médias dos índices de diversidade dos tratamentos que mantiveram a cobertura (MT, CPCV, CPSV) novamente se apresentaram estatisticamente semelhantes e superiores aos demais. Porém, na coleta anterior, SPSV $(H=0,3786)$ foi estatisticamente superior à $\operatorname{SPCV}(H=0,1072)$.

Houve flutuação da diversidade ao longo do ano apenas no tratamento SPSV. Nota-se que de novembro (2007) a maio (2008) ocorreu queda da diversidade, ocorrendo sua recuperação no mês de julho e igualdade aos outros tratamentos em setembro. Pela média geral do ano, pode-se concluir que não houve diferença estatística para a diversidade entre os tratamentos.

A Tabela 4 fornece os valores encontrados para a equitabilidade encontrada nos tratamentos ao longo do ano. Cabe lembrar que o valor do índice de Pielou (e) varia de 0 a 1 , sendo que quanto menor o índice maior será a dominância por poucos grupos.

Tabela 4. Equitabilidade da macrofauna edáfica (índices de Pielou) nas áreas da usina Alto Alegre, município de Colorado - Paraná, ao longo das seis épocas de coleta de novembro 2007 a setembro $2008^{(1)}$.

\begin{tabular}{llllllll}
\hline Tratamentos & \multicolumn{1}{c}{ Novembro $^{\text {ns }}$} & \multicolumn{1}{c}{ Janeiro $^{\text {ns }}$} & \multicolumn{1}{c}{ Março $^{\text {ns }}$} & \multicolumn{1}{c}{ Maio } & \multicolumn{1}{c}{ Julho } & \multicolumn{1}{c}{ Setembro } & Média $^{\text {ns }}$ \\
\cline { 2 - 7 } CPCV $^{\text {ns }}$ & 0,0936 & 0,2710 & 0,1531 & $0,2027 \mathrm{AB}$ & $0,2756 \mathrm{AB}$ & 0,3216 & 0,2196 \\
CPSV $^{\text {ns }}$ & 0,1967 & 0,1447 & 0,1161 & $0,1302 \mathrm{AB}$ & $0,2820 \mathrm{AB}$ & 0,3810 & 0,2085 \\
SPCV $^{\text {ns }}$ & 0,2131 & 0,1857 & 0,1620 & $0,0781 \mathrm{BC}$ & $0,0641 \mathrm{C}$ & 0,2632 & 0,1610 \\
$\mathrm{SPSV}$ & $0,1767 \mathrm{a}$ & $0,0605 \mathrm{~b}$ & $0,0360 \mathrm{~b}$ & $0,0181 \mathrm{C} \mathrm{b}$ & $0,2264 \mathrm{~B}$ a & $0,2191 \mathrm{a}$ & 0,1228 \\
MT $^{\text {ns }}$ & 0,1904 & 0,2138 & 0,3112 & $0,2388 \mathrm{~A}$ & $0,3828 \mathrm{~A}$ & 0,2619 & 0,2665 \\
\hline Média $^{\text {ns }}$ & 0,1741 & 0,1751 & 0,1557 & 0,1336 & 0,2462 & 0,2894 & \\
\hline
\end{tabular}

${ }^{(1)}$ Médias de cinco repetições. Análise sobre dados transformados por raiz $(x+0,5)$; valores na mesma época seguidos de letras iguais (maiúsculas) não se diferenciaram significativamente pelo teste de Duncan a 5\%. Valores no mesmo tratamento seguidos de letras iguais (minúscula) não se diferenciaram significativamente pelo teste de Duncan a 5\%. CPCV: com palha e com vinhaça; CPSV: com palha e sem vinhaça; SPCV: sem palha e com vinhaça; SPSV: sem palha e sem vinhaça; MT: mata; ns: não significativo.

Independentedotratamentohouvepredominância de poucos grupos, o que gerou índices baixos para todas as épocas. Entre os tratamentos houve diferença significativa somente nos meses de maio e julho, que revelaram maiores valores do índice de Pielou para áreas onde se manteve a cobertura do solo. Quando avaliada a flutuação da equitabilidade, ocorreu diferença significativa apenas em SPSV demonstrando maior sensibilidade deste ambiente. De acordo com Lourente et al. (2007) condições edafoclimáticas desfavoráveis têm efeitos negativos nas populações da macrofauna edáfica, as quais podem ter ocorrido nos meses em questão, já que nesta época do ano é comum a ocorrência de menores médias pluviométricas na região.

$\mathrm{Na}$ Tabela 5 estão expostas as médias do número de grupos (riqueza) encontrados no experimento. Nos meses de novembro (2007) e setembro (2008) não ocorreram variações estatísticas significativas da riqueza entre os tratamentos.

Em janeiro, os tratamentos $\mathrm{MT}$ e $\mathrm{CPCV}$ mostraram-se estatisticamente semelhantes (6,2 e 4 grupos), sendo seguidos por SPCV e CPSV 
(2,6 e 2,2 grupos), sendo SPSV com apenas 1,2 grupo, o tratamento com menor riqueza da fauna do solo, indicando que a interação da palhada, com a aplicação de vinhaça, proporcionaram melhores condições ao desenvolvimento de organismos edáficos em áreas de cana-de-açúcar, sendo estes resultados estendidos de março à julho.

Tabela 5. Riqueza da macrofauna edáfica nas áreas da usina Alto Alegre, município de Colorado - Paraná, ao longo das seis épocas de coleta de novembro 2007 a setembro $2008^{(1)}$.

\begin{tabular}{llllllll}
\hline Tratamentos & \multicolumn{1}{c}{ Novembro $^{\text {ns }}$} & \multicolumn{1}{c}{ Janeiro } & \multicolumn{1}{c}{ Março } & \multicolumn{1}{c}{ Maio } & Julho & \multicolumn{1}{c}{ Setembro } & Média \\
\cline { 2 - 7 } CPCV & $2,4 \mathrm{~b}$ & $4,0 \mathrm{AB} \mathrm{ab}$ & $2,4 \mathrm{AB} \mathrm{b}$ & $3,4 \mathrm{AB}$ ab & $5,4 \mathrm{AB}$ a & $5,4 \mathrm{a}$ & $3,8 \mathrm{~B}$ \\
$\mathrm{CPSV}$ & $2,8 \mathrm{ab}$ & $2,2 \mathrm{BC} \mathrm{bc}$ & $2,0 \mathrm{~B} \mathrm{c}$ & $2,4 \mathrm{~B} \mathrm{bc}$ & $4,0 \mathrm{~B} \mathrm{ab}$ & $5,4 \mathrm{a}$ & $3,1 \mathrm{~B}$ \\
SPCV & $2,6 \mathrm{~b}$ & $2,6 \mathrm{BC} \mathrm{b}$ & $2,2 \mathrm{AB} \mathrm{b}$ & $2,2 \mathrm{~B} \mathrm{~b}$ & $2,0 \mathrm{C} \mathrm{b}$ & $5,4 \mathrm{a}$ & $2,8 \mathrm{~B}$ \\
SPSV & $5,6 \mathrm{a}$ & $1,2 \mathrm{C} \mathrm{b}$ & $1,0 \mathrm{~B} \mathrm{~b}$ & $0,8 \mathrm{C} \mathrm{b}$ & $3,6 \mathrm{~B} \mathrm{a}$ & $3,4 \mathrm{a}$ & $2,6 \mathrm{~B}$ \\
MT & $5,8 \mathrm{a}$ & $6,2 \mathrm{~A} \mathrm{a}$ & $4,6 \mathrm{~A} \mathrm{a}$ & $4,2 \mathrm{~A} \mathrm{a}$ & $6,6 \mathrm{~A} \mathrm{a}$ & $5,8 \mathrm{a}$ & $5,5 \mathrm{~A}$ \\
\hline Média & $3,84 \mathrm{a}$ & $3,24 \mathrm{a}$ & $2,44 \mathrm{a}$ & $2,6 \mathrm{a}$ & $4,32 \mathrm{a}$ & $5,08 \mathrm{a}$ & \\
\hline
\end{tabular}

${ }^{(1)}$ Médias de cinco repetições. Análise sobre dados transformados por raiz $(x+0,5)$; valores na mesma época seguidos de letras iguais (maiúsculas) não se diferenciaram significativamente pelo teste de Duncan a 5\%. Valores no mesmo tratamento seguidos de letras iguais (minúscula) não se diferenciaram significativamente pelo teste de Duncan a 5\%. CPCV: com palha e com vinhaça; CPSV: com palha e sem vinhaça; SPCV: sem palha e com vinhaça; SPSV: sem palha e sem vinhaça; MT: mata; ns: não significativo.

No mês de julho MT apresentou média de riqueza significativamente superior, porém $\mathrm{CPCV}$ revelou valores intermediários entre os índices de riqueza encontrados na mata e os tratamentos CPSV e SPSV. O tratamento SPCV foi estatisticamente inferior a todos os outros nesta coleta. Os resultados obtidos corroboram com os de Pinheiro, Santos e Garay (1996), no qual a cana colhida sem queima em comparação com a colhida com uso do fogo apresentou em duas épocas avaliadas maior riqueza da fauna do solo e ainda com Pimentel et al. (2006) cujos valores para riqueza da macrofauna foram superiores em floresta do que em diferentes sistemas de manejo.

Os dados obtidos e os autores citados apontam que a manutenção da palha sobre o solo contribui para maiores índices de riqueza. Em relação à flutuação da riqueza no ano, a área de mata foi a única a apresentar dados constantes, sugerindo estabilidade da fauna, o que mantém a ocupação dos nichos de forma mais constante.
A Análise de Componentes Principais (Figura 3) explicou a variabilidade dos dados em $36,1 \%$ pela componente principal $1(\mathrm{CP}), 21,7 \%$ pela pela $\mathrm{CP} 2$ e $15,0 \%$ pela CP 3 (não representado), totalizando $72,7 \%$ da variabilidade total. De acordo com Ter Braak e Smilaurer (2002) a soma dos três primeiros eixos acima de $70 \%$ indicam boa explicação para a variabilidade dos dados. A CP 1 separou os tratamentos MT e CPCV à direita e o restantes dos tratamentos à esquerda, evidenciando o efeito palha. O tratamento MT ficou relacionado aos grupos Isoptera, Coleoptera, Outros Invertebrados e Oligochaetas. O tratamentos CPCV, relacionouse à Formicidae, Araneae, Outros Invertebrados e Coleoptera. Concordando com estes resultados A CP 2 evidencia o efeito da vinhaça na separação dos grupos nos quadrantes. CPSV ficou associado aos grupos Formicidae e Hemiptera. O tratamento SPSV caracterizou-se por apresentar as menores correlações com os organismos edáficos, apresentado pouca correlação apenas com o grupo Hemiptera. 
Figura 3. Análise de componentes principais para comunidade edáfica média encontrada de novembro de 2007 à setembro 2008 nas áreas da usina Alto Alegre, Colorado - Paraná.

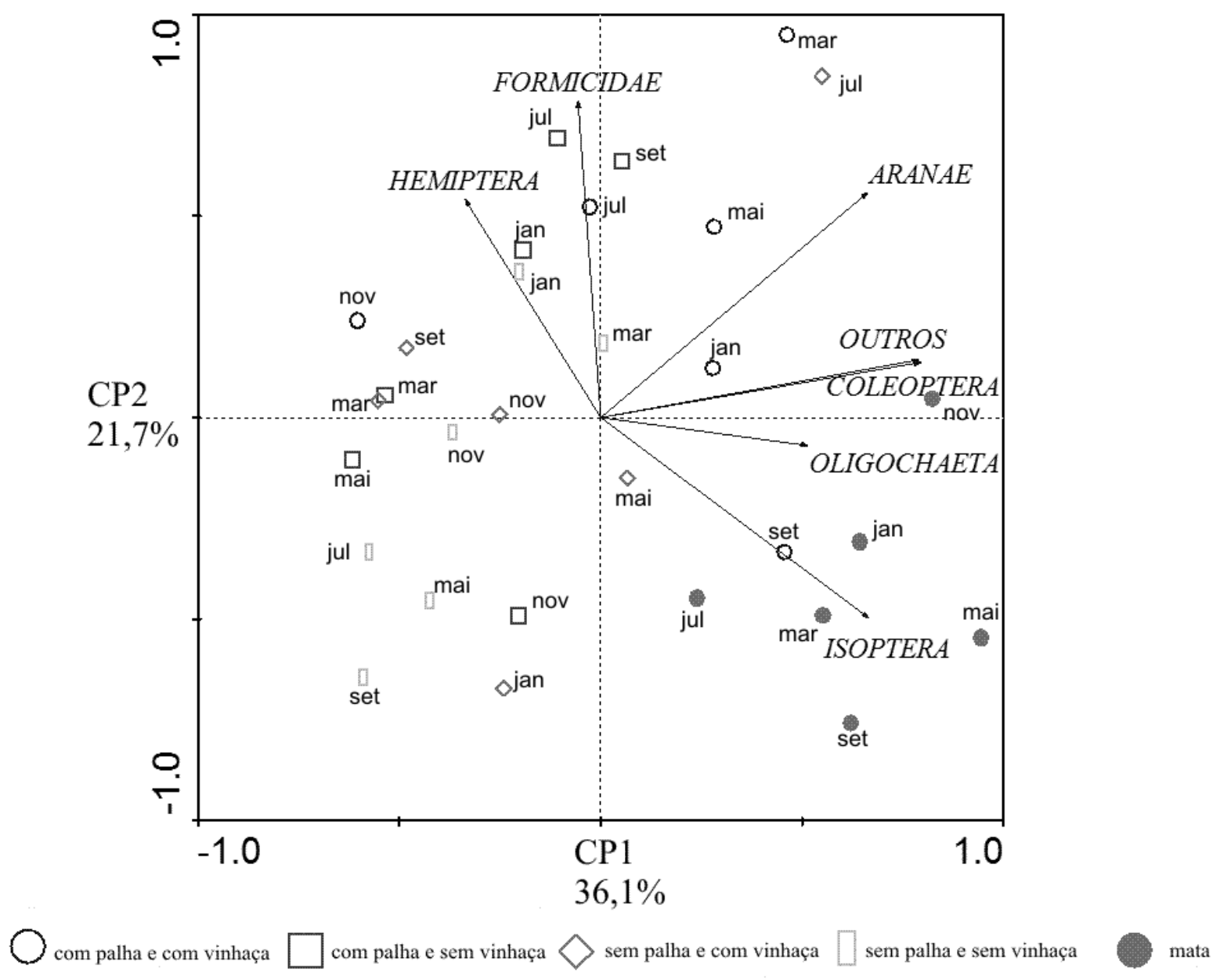

Fonte: Elaboração dos autores.

\section{Conclusões}

Os grupos taxonômicos que caracterizam áreas de cana-de-açúcar são: Formicidae, Hemiptera, Araneae, Coleoptera, Oligochaeta.

Os grupos taxonômicos que caracterizam área de mata são: Oligochaeta, Coleoptera, Outros Invertebrados e principalmente Isoptera.

A colheita mecanizada (manutenção da palhada) proporciona benefícios à macrofauna edáfica em áreas de cana-de-açúcar, tendo índices de diversidade, equitabilidade e riqueza de grupos mais elevados do que áreas onde não se mantém a liteira.
Área de mata apresenta maiores índices de diversidade, equitabilidade e riqueza de grupos comparativamente com áreas com cana-de-açúcar.

A não aplicação da vinhaça ou manutenção da cobertura do solo acarreta maiores flutuações da diversidade, equitabilidade e riqueza em áreas de cana-de-açúcar ao longo do tempo.

\section{Agradecimentos}

À Usina de Açúcar e Álcool Alto Alegre S. A. - Unidade Junqueira, por todo apoio prestado à realização deste trabalho. 


\section{Referências}

ANDERSON, J. M.; INGRAM, J. S. I. Soil fauna. In: (Ed.). Tropical soil biological and fertility: a handbook of methods. 2. ed. Wallingford: C.A.B. International, 1993. p. 44-46.

ANDERSON, J. M.; BENJAMIN, D. H.; MÜLLER, W.; GRIFFETHS, A. D. Using ants as bioindicators in land management simplifying assessment of ant community responses. Journal of Applied Ecology, Beijing, v. 39, n. 1, p. 8-17, 2002.

AZANIA, A. A. P. M.; MARQUES, M. O.; PAVANI, M. C. M. D.; AZANIA, C. A. M. Germinação de sementes de Sida rhombifolia e Brachiaria decumbens influenciada por vinhaça, flegmaça e óleo de fúsel. Planta Daninha, Viçosa, MG, v. 21, n. 3, p. 443-449, 2003.

BARETTA, D.; SANTOS, J. C. P.; MAFRA, Á. L.; WILDNER, L. do P.; MIQUELLUTI, D. J. Fauna edáfica avaliada por armadilhas de catação manual afetada pelo manejo do solo na região oeste catarinense. Revista de Ciências Agroveterinárias, Lages, v. 2, n. 1, p. 97-106, 2003.

BIANCHI, M. O.; AQUINO, A. M.; ALMEIDA, E. Distribuição vertical da macrofauna do solo em várias safras do Milho agroecológico em área do produtor familiar. In: CONGRESSO BRASILEIRO DE CIÊNCIA DO SOLO, 31.; CONGRESSO BRASILEIRO DE CIÊNCIA DO SOLO-CONQUISTAS E DESAFIOS DA CIÊNCIA DO SOLO BRASILEIRA, 31., 2007, Gramado, RS. Anais... Gramado, RS: Sociedade Brasileira de Ciência do Solo, 2007. CD-ROM.

CANELLAS, L. P.; BALDOTTO, M. A.; BUSATO, J. G.; MARCIANO, C. R.; MENEZES, S. C.; SILVA, M. N da; RUMJANEK, V. M.; VELLOSO, A. C. X.; SIMÕES, M. L.; MARTIN-NETO, L. M. Estoque e qualidade da matéria orgânica de um solo cultivado com cana-deaçúcar por longo tempo. Revista Brasileira de Ciência do Solo, Viçosa, MG, v. 31, n. 2, p. 331-340, 2007.

CANELLAS, L. P.; VELLOSO, A. C. X.; MARCIANO, C. R.; RAMALHO, J. F. G. P.; RUMJANEK, V. M.; REZENDE, C. E.; SANTOS, G. A. Propriedades químicas de um cambissolo cultivado com cana-deaçúcar, com preservação do palhiço e adição de vinhaça por longo tempo. Revista Brasileira de Ciência do Solo, Viçosa, MG, v. 27, n. 5, p. 935-944. 2003.

COMPANHIA NACIONAL DE ABASTECIMENTO CONAB. Estimativas de safras. Revista Indicadores da Agropecuária, Brasília, ano XIV, n. 9, p. 8, 2005.

COSTA, E. A.; GOEDERT, W. J.; SOUSA, D. M. G de. Qualidade de solo submetido a sistemas de cultivo com preparo convencional e plantio direto. Pesquisa
Agropecuária Brasileira, Brasília, v. 41, n. 7, p. 11851191. 2006.

DLAMINI, T. C.; HAYNES, R. J. Influence of agricultural land use on the size and composition of earthworm communities in northern KwaZulu-Natal, South Africa. Applied Soil Ecology, Amsterdam, v. 27, n. 1, p. 77-88, 2004.

EMPRESA BRASILEIRA DE PESQUISA AGROPECUÁRIA - EMBRAPA. Sistema brasileiro de classificação de solos. 2. ed. Rio de Janeiro: Embrapa Solos, 2006. 306 p.

FLÓREZ, E. D. Comunidades de aramas de la región Pacífica del departamento del Valle del Cauca, Colômbia. Revista Colombiana de Entomologia, Bogotá, v. 26, n. 4, p. 77-81, 2000.

GÓMES, J.; RODRÍGUEZ, O. Effects of vinasse on sugarcane (Saccharum officinarum) productivity. Revista da la Faculdade de Agronomia LUZ, Maracaibo, v. 17, n. 4, p. 318-326, 2000.

INSTITUTO AGRONÔMICO DO PARANÁ - IAPAR. Cartas climáticas do Estado do Paraná 1994. Londrina: IAPAR, 1994. 49 p. (IAPAR, Documento, 18).

LIMA, S. S de; AQUINO, A. M de; LEITE, L. F. C.; SILVA, P. H. S. da; CASTRO, A. A. J. F.; OLIVEIRA, $\mathrm{F}$ das C. Diversidade da macrofauna edáfica em agroflorestas de diferentes estádios sucessionais. Revista Brasileira de Agroecologia, Cruz Alta, RS, v. 2, n. 2, p. 1029-1033, 2007.

LOURENTE, E. R. P.; SILVA, R. F. da; SILVA, D. A. da; MARCHETTI, M. E.; MERCANTE, F. M. Diferentes sistemas de manejo do solo e seus efeitos sobre a Macrofauna Edáfica. In: CONGRESSO BRASILEIRO DE CIÊNCIA DO SOLO, 31; CONGRESSO BRASILEIRO DE CIÊNCIA DO SOLO-CONQUISTAS E DESAFIOS DA CIÊNCIA DO SOLO BRASILEIRA, 31., 2007, Gramado, RS. Anais... Gramado, RS: Sociedade Brasileira de Ciência do Solo, 2007. p. 1-4.

MOÇO, M. K.; GAMA-RODRIGUES, E. F.; GAMARODRIGUES, A. C.; CORREIA, M. E. Caracterização da fauna edáfica em diferentes coberturas vegetais na região norte fluminense. Revista Brasileira de Ciência do Solo, Viçosa, MG, v. 29, n. 4, p. 555-564, 2005.

NARDI, C. Percevejos castanhos (Hemiptera, Cydnidae, Scaptocoris): aspectos morfológicos, ecológicos e comportamentais. 2005. Dissertação (Mestrado em Ciências) - Escola Superior de Agricultura Luiz de Queiroz, Piracicaba.

NUNES, D. H.; PASINI, A.; BENITO, N. P.; BROWN, G. G. Earthworm diversity in four land use systems in the region of Jaguapitã, Paraná state, Brazil. Caribbean 
Journal of Science, Mayagüez, Puerto Rico, v. 42, n. 3, p. 331-338, 2006.

PIMENTEL, M.; AQUINO, A.; CORREIA, M.; COSTA, J.; RICCI, M.; DE-POLLI, H. Atributos biológicos do solo sob manejo orgânico de cafeeiro, pastagem e floresta em região do Médio Paraíba Fluminense-RJ. Coffee Science, Lavras, MG, v. 1, n. 2, p. 83-93, 2006.

PINHEIRO, L. B. A.; SANTOS, G. de A.; GARAY, I. E. Efeito da queima da palhada da cana-de-açúcar na população de macroartrópodos edáficos. In: CONGRESSO LATINO AMERICANO DE CIÊNCIA DO SOLO, 13., 1996, Águas de Lindóia, SP. Resumos... Águas de Lindóia, SP: USP/SLCS/SBCS, 1996. CDROM.

RAZAFIMBELO, T.; BARTHÈS, B.; LARROUYLARRÉ, M. C.; DE LUCA, E. F.; LAURENT, J. Y.; CERRI, C. C.; FELLER, C. Effect of sugarcane residue management (mulching versus burning) on organic matter in a clayey Oxisol from southern Brazil. Agriculture, Ecosystems and Environment, Zürich, Switzerland, v. 115, n. 4, p. 285-289, 2006.

RIBEIRO, H. Queimadas de cana-de-açúcar no Brasil: efeitos à saúde respiratória. Revista Saúde Pública, São Paulo, v. 42, n. 2, p. 370-376, 2008.

SANOMIYA, L. T.; ASSIS, L. C.; OLIVEIRA, J. A. de; NAHAS, E. Mineralización de la palja de caña de azúcar en suelo adicionado com viñaza (subproducto de la industria del alcohol de caña de azúcar) y fertilizante nitrogenado. Agricultura Técnica, Chile, v. 66, n. 1, p. 90-97, 2006.

SILVA, R. F. da; MERCANTE, F. M. ; AQUINO, A. M. ; GUIMARAES, M. F. Engenheiros ecológicos e atributos químicos do solo sob diferentes sistemas de manejo. In: FERTBIO, 2006, Bonito, MS. Anais... Bonito, MS: Sociedade Brasileira de Ciência do Solo, 2006. CDROM.

SOUZA, Z. M. de; PAIXÃO, A. C. S.; PRADO, R de M.; CESARIN, L. G.; SOUZA, S. R de. Manejo de palhada de cana colhida sem queima, produtividade do canavial e qualidade do caldo. Ciência Rural, Santa Maria, v. 35, n. 5, p. 1062-1068, set./out. 2005.

SPOSITO, G.; ZABEL, A. The assessment of soil quality. Geoderma, Amsterdam, v. 114, n. 3/4, p. 143-144, 2003.

TER BRAAK, C. J. F.; SMILAURER, P. CANOCO reference manual and canodraw for windows user's guide: software for canonical community ordination (version 4.5). Microcomputer power. Ithaca, NY: [s.n], 2002.

URIARTE, M.; YACKULIC, C. B.; COOPER, T.; FLYNN, D.; CORTES, M.; CRK, T.; CULLMAN, G.; McGINTY, M.; SIRCELY, J. Expansion of sugarcane production in São Paulo, Brazil: implications for fire occurrence and respiratory health. Agriculture, Ecosystems and Environment, Zürich, Switzerland, v. 132, n. 1, p. 48-56, 2009. 
